Journal of Case Reports 2018;8(4):282-285

\title{
Sodium Valproate as a Probable Cause of Gut Gangrene: Report of Two Unusual Cases
}

\author{
Jitendra Singh, Satish Dalal, Chisel Bhatia, Amiraj Singh, Jagatpal Beniwal \\ Department of General Surgery, Pt. B. D. Sharma Postgraduate Institute of Medical Sciences (P.G.I.M.S.), Rohtak-124001, \\ Haryana, India.
}

\section{Corresponding Author: \\ Dr. Jitendra Singh \\ Email: jitenderasingh742@gmail.com}

This is an Open Access article distributed under the terms of the Creative Commons Attribution License (creativecommons.org/ licenses/by/3.0).

Received Accepted Published

\begin{abstract}
Background: Gangrenous gut is a highly fatal condition most often caused by mesenteric ischemia or intestinal obstruction and requires emergency surgical intervention. There is no definite evidence about role of anti-epileptics as a cause of gut gangrene. Case Report: We encountered two patients who presented with gangrenous bowel and both patients had history of treatment of sodium valproate. There was no other evident cause for gut gangrene in these patients. Conclusion: Hence, with this case report we discuss and emphasize that sodium valproate can be a probable cause for gut gangrene in patients who are undergoing treatment with this agent.
\end{abstract}

Keywords: Anticonvulsants, Gangrene, Intestinal Obstruction, Mesenteric Ischemia, Valproic Acid.

\section{Introduction}

Gangrenous gut is a life threatening condition, most often caused by mesenteric ischemia or intestinal obstruction due to hernias and adhesions. Mortality rate in gangrenous gut is as high as one third of the total patients and mesenteric ischemia is the leading cause of deaths in these patients [1]. Mesenteric ischemia caused by inadequate vascular supply to the gut can be acute or chronic. Infarction of the gut as a consequence of ischemia can also occur due to various drugs having basic patho-physiology either by vasopressor action or enhancing thrombus formation. Thus, drugs and various other hyper-coagulable states make a person more vulnerable to mesenteric ischemia leading to gut gangrene.

There are few references in the literature where antiepileptic drugs like sodium valproate, phenytoin, carbamazepine lead to derangement of the lipid and coagulation profile, which in turn leads to a hyper-coagulable state and a higher risk of cardiovascular complications. Therefore, the role of anti-epileptics as a possible cause for gangrenous bowel cannot be ignored. Here we present two cases in which patients were found having gangrenous gut intra-operatively and have consumed sodium valproate for some duration prior to the episode.

\section{Case Report}

\section{Case 1}

A 33 year old, male presented in emergency department with complaints of right iliac fossa pain, loose stools and vomiting for three days. The patient was a known case of seizure disorder for which he was taking sodium valproate tablets on a daily basis for last one year. On examination, abdomen was soft with tenderness over right iliac fossa. His hemoglobin was $11.2 \mathrm{gm} / \mathrm{dL}$ and total leukocyte counts were $14,000 / \mathrm{mm}^{3}$. Ultrasonography of the abdomen was suggestive of inflammed appendix with minimal fluid present 
in pelvis. The patient was diagnosed as a case of burst appendix. The patient underwent emergency exploratory laparotomy with the following intraoperative finding i.e. around $100 \mathrm{cc}$ of purulent fluid present in peritoneal cavity; pus flakes present over gut and parities in right iliac fossa; gangrenous appendix, cecum and proximal ascending colon with cecal perforation; dense adhesion present between omentum, gangrenous gut and parities. Resection of gangrenous gut with end ileostomy with appendicectomy was performed. Patient had an uneventful post-operative period and was discharged on post-operative day thirteenth. Ileostomy closure was performed two months later on elective basis.

\section{Case 2}

A 24 year old, male presented in emergency department with complaints of pain abdomen localizing to right lower quadrant and vomiting for six days; associated with fever and non passage of flatus and stool for two days. He was on antiepileptic, sodium valproate for past six months prescribed by private hospital and is undergoing investigations for the demyelinating plaques present over $\mathrm{C} 3, \mathrm{C} 4$. Abdominal examination revealed a soft and tender abdomen localizing to right iliac fossa; a palpable lump measuring approximately $4 \times 3 \mathrm{~cm}$ at right lower quadrant. Routine laboratory investigations were sent and his hemoglobin was found to be $8.9 \mathrm{gm} / \mathrm{dL}$ and total leukocyte counts were $13,000 / \mathrm{mm}^{3}$. Ultrasonography of the abdomen was suggestive of burst appendix. Patient underwent emergency exploratory laparotomy with resection of gangrenous gut with end ileostomy. Intra-operatively, around $200 \mathrm{cc}$ of purulent fluid present in peritoneal cavity; gangrenous cecum, ascending colon with omentum adherent to them; terminal ileum was found edematous and adherent to gangrenous colon; pus flakes were present over liver, omentum and ileum. After an uneventful post-operative hospital stay patient was discharged under stable conditions and planned for ileostomy closure two months later.

\section{Discussion}

Gangrene is the condition in which the vascular supply to the organ get compromised and as a result of which tissue death occurs. Similarly, the conditions which cause low perfusion of the bowel leads to gut gangrene which is one of the surgical emergencies with poor prognosis if management is delayed. The timely use of diagnostic and therapeutic methods to quickly restore blood flow to the bowel is the key to reduce the high mortality associated with this condition $[2,5]$.

The gut normally receives 20 to $25 \%$ of cardiac output, thus making the gut very sensitive to the effects of decreased perfusion. Three major vessels serve the abdominal contents: celiac trunk, superior mesenteric artery, and inferior mesenteric artery. Although the gut can survive $75 \%$ reduction in blood flow for up to 12 hours without significant injury [6], irreversible bowel damage occurs within six hours of complete vascular occlusion [7].

According to Barnett et al. the overall mortality rate was $37 \%$ in 151 cases of gangrenous bowel. Figures obtained in his study reveal mesenteric ischemia as a cause of gut gangrene to be a dangerous condition with a high mortality rate of $74 \%$. Mortality rate were $20 \%$ and $23 \%$ if gut gangrene is due to hernia and adhesions respectively [1].

The four major causes of acute mesenteric ischemia are arterial embolus, arterial thrombosis, mesenteric venous thrombosis, non-occlusive mesenteric ischemia. However, no etiology may be found in approximately $15 \%$ patients [8-10]. Acute mesenteric ischemia can be secondarily due to mechanical obstruction like in cases of strangulated hernia, intussusception, volvulus etc. Occasionally the blood supply to the gut may get compromised due to injury to the superior mesenteric artery in blunt trauma abdomen. Tumor compression, aortic dissection and post-angiography are other rare reported cases causing ischemia of gut. 
Various drugs like large doses of vasopressors alone can cause bowel ischaemia by low perfusion mechanism. Several case reports link cardiotoxins, such as digitalis, in combination with furosemide-induced fluid depletion, calciumchannel blockers, organophosphates, ergotamine, phenobarbital, ethylene glycol or tricyclic antidepressants to non-obstructive mesenteric ischemia in intensive care unit patients [11].

The study conducted by Katsiki et al. revealed that antiepileptic drugs may affect the biochemical parameters and can derange the emerging predictors of vascular risk [12]. This is the well known fact that high levels of homocysteine are associated with hyper-coagulability [13]. Several studies have reported sodium valproate, one of the extensively used antiepileptic drug has been associated with elevated homocysteine levels [14-16]. It is also found to be associated with increased levels of lipoprotein (A) in various studies conducted on children [17-19]. Here, in our clinical setup we are encountered with two such cases in which intra-operative gangrenous bowel was present and their detailed history revealed that both of the patients were on sodium valproate. Thus considering sodium valproate to be a drug causing hyper-coagulable state there lies a possibility that sodium valproate may compromise the splanchnic perforation leading to mesenteric ischaemia and gangrenous bowel.

\section{Conclusion}

We encountered two cases who consumed sodium valproate for some reason. Both these cases were found having a gangrenous bowel and the fact that antiepileptics can derange the lipid and coagulation profile, a high degree of suspicion is necessary in view of antiepileptics consumption in the patients presenting with symptoms of gangrenous bowel so as to make provisional diagnosis more correct, thus preventing the delay in management. Also, further studies must be conducted to found relation between sodium valproate and gangrenous bowel so that we can have extra precaution while using sodium valproate and consumers must be told about the symptoms of gangrenous gut before oral intake so as to prevent the delay in management thus better survival rate.

Contributors: JS, AS: manuscript drafting, literature search and patient management; SD, CB, JB: manuscript editing, critical inputs into the manuscript and patient management. JS will act as guarantor of the study. All authors approved the final version of this manuscript.

Funding: None; Competing interests: None stated.

\section{References}

1. Barnett WO, Petro AB, Williamson JW. A current appraisal of problems with gangrenous bowel. Annals of Surgery. 1976;183:653-659.

2. Martinez JP, Hogan GJ. Mesentric ischemia. Surg Clin North Am. 2004;22:909-928.

3. Wilcox MG, Howard TJ, Plaskon LA, Unthank JL, Madura JA. Current theories of pathogenesis and treatment of nonocclusive mesenteric ischemia. Dig Dis Sci. 1995;40:709-716.

4. Berney T, Morales M, Broquet PE, Mentha G, Morel P. Risk factors influencing the outcome of portal and mesenteric vein thrombosis. Hepatogastroenterology. 1998;45:2275-2281.

5. Vicente DC, Kazmers A. Acute mesenteric ischemia. Curr Opin Cardiol. 1999; 14:453-458.

6. Boley SJ, Brandt LJ, Veith FJ. Ischemic disorders of the intestine. Curr Probl Surg. 1978;15:1-85.

7. Chin CJ, McArdle AH, Brown R, Scott HJ, Gurd FN. Intestinal mucosal lesion in low-flow states. A morphological, hemodynamic and metabolic reappraisal. Arch Surg. 1970;101:478-483.

8. Lock G. Acute intestinal ischaemia. Best Pract Res Clin Gastroeterol. 2001;15:83-98.

9. Mansour MA. Management of acute mesenteric ischemia. Arch Surg. 1999;134:328-330.

10. Dang CV, Su M, Nishijima DK. Acute Mesenteric Ischemia. eMedicine.com, Inc. 2018. Available at: https://emedicine.medscape.com/article/189146overview. Accessed on September $4^{\text {th }} 2018$.

11. Tilsed JVT, Casamassima A, Kurihara H, Mariani D, Martinez I, Pereira J, et al. ESTES guidelines: acute mesenteric ischaemia. European Journal of Trauma and Emergency Surgery. 2016;42:253-270.

12. Mohan IV, Jagroop IA, Mikhailidis DP, Stansby GP. Homocysteine activates platelets in vitro. Clin Appl Thromb Hemost. 2008;14:8-18.

13. Kim DW, Lee SY, Shon YM, Kim JH. Effects of new antiepileptic drugs on circulatory markers for vascular 
risk in patients with newly diagnosed epilepsy. Epilepsia 2013;54:e146-149.

14. Apeland T, Mansoor MA, Strandjord RE. Antiepileptic drugs as independent predictors of plasma total homocysteine levels. Epilepsy Res. 2001;47:27-35.

15. Striano P, Gorgone G, Costa C, Ciampa C, Caccamo $\mathrm{D}$, Pisani LR, et al. Hyperhomocysteinemia in epileptic patients on new antiepileptic drugs. Epilepsia. 2010;51:274-279.

16. Castro-Gago M, Novo-Rodriguez MI, Blanco-Barca MO, Urisarri-Ruiz de Corta-zar A, Rodriguez-Garcia J, Rodriguez-Segade S, et al. Evolution of serum lipids and lipoprotein (a) levels in epileptic children treated with carbamazepine, valproic acid and phenobarbital. J Child Neurol. 2006;21:48-53.

17. Sonmez FM, Demir E, Orem A, Yildirmis S, Orhan F, Aslan A, et al. Effect of antiepileptic drugs on plasma lipids, lipoprotein (a) and liver enzymes. J Child Neurol. 2006;21:70-74.

18. Voudris KA, Attilakos A, Katsarou E, Drakatos A, Dimou S, Mastroyianni S, et al. Early and persistent increase in serum lipoprotein (a) concentrations in epileptic children treated with carbamazepine and sodium valproate monotherapy. Epilepsy Res. 2006;70:211-217. 